\title{
A NOTE ON A PROBLEM OF ROBINSON
}

\author{
KENT PEARCE
}

\begin{abstract}
Let $s$ be the usual class of univalent analytic functions on $|z|<1$ normalized by $f(0)=0$ and $f^{\prime}(0)=1$. Let $E$ be the linear operator on $\$$ given by $E^{\prime} f={ }_{2}^{\prime}(z f)^{\prime}$ and let $r_{s}$, be the minimum radius of starlikeness of $\varrho f$ for $f$ in $\Omega$. In 1947 R. M. Robinson initiated the study of properties of $\mathcal{L}$ acting on $\mathcal{S}$ when he showed that $r_{s t}>$.38. Later, in 1975, R. W. Barnard gave an example which showed $r_{s_{1}}<.445$. It is shown here, using a distortion theorem and Jenkin's region of variability for $z f^{\prime}(z) / f(z), f$ in $s$. that $r_{s_{t}}>.435$. Also, a simple example, a close-to-convex half-line mapping, is given which again shows $r_{s_{t}}<.445$.
\end{abstract}

Introduction. Let $\mathscr{Q})$ denote the open unit disk $\{z|| z \mid<1\}$ and let $Q$ be the class of analytic functions on $Q \mathcal{D}$. Let $S$ be the subclass of $\mathcal{Q}$ of univalent functions $f$ normalized by $f(0)=0$ and $f^{\prime}(0)=1$. Let $\delta t, \mathcal{K}$ and $\mathcal{C}$ denote the usual subclasses of $\varsigma$ of starlike, convex and close-to-convex functions, respectively. For $0 \leqslant \alpha<1$, let $\delta t(\alpha)$ be the subclass of $\delta t$ of starlike functions of order $\alpha$. Finally, let $R$ denote the subclass of $S$ of functions $f$ satisfying $\operatorname{Re} f^{\prime}(z)>0, z \in \mathscr{D}$. For any compact subclass $\mathscr{X}$ of $\mathcal{Q}$ (possibly a singleton) let $r_{\S}(\mathfrak{X})\left[r_{s_{t}}(\mathfrak{X})\right.$, etc.] denote the minimum radius of univalence [starlikeness, etc.] over $f$ in $\mathcal{X}$.

Let $\mathcal{L}$ be the linear operator on $\mathcal{Q}$ given by $\mathcal{L} f=\frac{1}{2}(z f)^{\prime}$. The study of the extent to

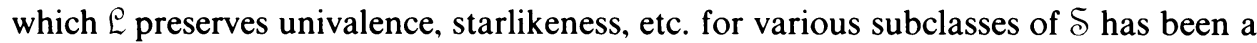
recurrent theme in the literature. R. M. Robinson [12, p. 18] initiated the study in 1947 in a paper where he showed that $r_{\varsigma_{t}}[\mathcal{L}(\mathcal{S})]>.38$. That implied, of course, $r_{\S}[\mathcal{L}(\varsigma)]>.38$. He conjectured then that $r_{\S}[\mathcal{L}(\varsigma)]=\frac{1}{2}$. (For the Koebe function $k$ it is easily seen that $r_{s}[\mathcal{E} k]=r_{s_{t}}[\mathcal{E} k]=\frac{1}{2}$.) Little or no progress was made directly on the study of $E$ following Robinson's work until 1966 when A. E. Livingston [10] proposed a shift for the setting of the problem from the full class $\mathcal{S}$. He showed that for each of the subclasses $\varsigma t, \mathscr{K}$ and $\mathcal{C}$, a form of Robinson's $\frac{1}{2}$ conjecture does hold, i.e.,

$$
r_{S t}\left[\mathcal{L}\left(S_{t}\right)\right]=r_{K}[\mathcal{L}(K)]=r_{\mathrm{e}}[\mathcal{L}(\mathcal{C})]=1 / 2 .
$$

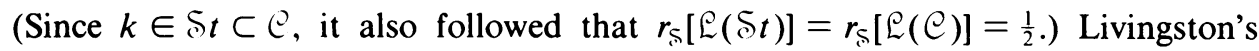
work renewed interest in the study of $E$. A series of papers [11,9,3 and 2] appeared in which the values of $r_{\delta t(\beta)}[\mathcal{L}(\delta t(\alpha))]$ were determined for various ranges of the parameters $\alpha$ and $\beta$. In [1 and 7] the values of $r_{\delta_{t}}[\mathcal{L}(\mathcal{X})]$ were found for several subclasses $\mathcal{X}$ of $\delta t$ given by coefficient restrictions. Also, in $[1,7,9$ and 2$]$ related

Received by the editors July 9, 1982. Presented to the AMS at the annual meeting at Denver, Colorado, on Wednesday, January 5, 1983.

1980 Mathematics Subject Classification. Primary 30C45.

Key words and phrases. Univalence, radius of starlikeness, Robinson's $\frac{1}{2}$ conjecture. 
results were given for various subclasses of $\mathscr{K}$ and $\mathcal{R}$. A number of techniques were used to approach these problems; however, with the introduction of convolution theory, a single approach was developed which could solve most of these problems [6].

For the class $\delta$, however, no improvements on Robinson's original work were made until 1975 when R. W. Barnard [4] gave an example which showed $r_{\varsigma_{t}}[\mathcal{L}(\mathcal{S})]<$ .445. A consequence of Barnard's example was that separate techniques would be

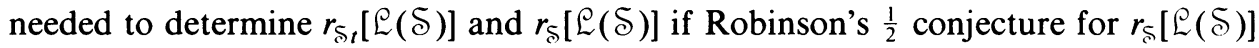
held. In 1978, applying the Grunsky inequalities to the univalence problem, Barnard [5] showed $.49<r_{\S}[\mathcal{L}(\mathcal{S})]$.

The best known estimates for $r_{\delta t}[\mathcal{L}(\delta)]$ have been the lower bound .38 from Robinson's original work and the upper bound .445 from Barnard's example. In this paper we will show, using an elementary distortion theorem and Jenkin's region of variability for $z f^{\prime}(z) / f(z), f$ in $\mathcal{S}$, that $r_{\delta t}[\mathcal{L}(\mathcal{S})]>.435$. We will also give a simple example (a function $f$ in $\mathcal{C}$ ) which again shows that $r_{S_{t}}[\mathcal{L}(\mathcal{S})]<.445$.

Lower bound. It is well known that a function $g$, analytic in $|z|<r$, is starlike (w.r.t. 0) in $|z|<r$ if and only if $\operatorname{Re} z g^{\prime}(z) / g(z)>0$ for $|z|<r$. If $g=\mathcal{L}(f)$, then

$$
\frac{z g^{\prime}(z)}{g(z)}=\frac{2+z f^{\prime \prime}(z) / f^{\prime}(z)}{1+f(z) /\left(z f^{\prime}(z)\right)} \text {. }
$$

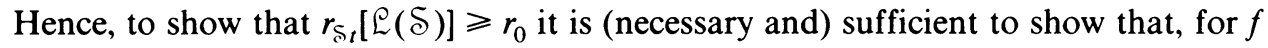
in $\delta$,

$$
\operatorname{Re} \frac{2+z f^{\prime \prime}(z) / f^{\prime}(z)}{1+f(z) /\left(z f^{\prime}(z)\right)}>0, \quad|z|<r_{0} .
$$

Let $f \in \mathcal{S}$ and $r=|z|$. It is well known that

$$
\left|\frac{z f^{\prime \prime}(z)}{f^{\prime}(z)}-\frac{2 r^{2}}{1-r^{2}}\right| \leqslant \frac{4 r}{1-r^{2}}
$$

which can be rewritten as

$$
\left|2+\frac{z f^{\prime \prime}(z)}{f^{\prime}(z)}-\frac{2}{1-r^{2}}\right| \leqslant \frac{4 r}{1-r^{2}} .
$$

If we divide both sides of $(1)$ by $1+f(z) /\left(z f^{\prime}(z)\right)$, we obtain

(2)

$$
\left|\frac{2+z f^{\prime \prime}(z) / f^{\prime}(z)}{1+f(z) /\left(z f^{\prime}(z)\right)}-\frac{2}{1-r^{2}} \frac{z f^{\prime}(z) / f(z)}{1+z f^{\prime}(z) / f(z)}\right| \leqslant \frac{4 r}{1-r^{2}}\left|\frac{z f^{\prime}(z) / f(z)}{1+z f^{\prime}(z) / f(z)}\right| \text {. }
$$

Let $W$ denote $z f^{\prime}(z) / f(z)$. Then (2) implies

$$
\operatorname{Re} \frac{2+z f^{\prime \prime}(z) / f^{\prime}(z)}{1+f(z) / z f^{\prime}(z)} \geqslant \operatorname{Re} \frac{2}{1-r^{2}} \frac{W}{1+W}-\frac{4 r}{1-r^{2}}\left|\frac{W}{1+W}\right| .
$$

J. A. Jenkins $[8$, p. 110] has shown that the region of variability for $W=$ $z f^{\prime}(z) / f(z), f$ in $\delta$, is exactly the set given by

$$
|\log W| \leqslant \log ((1+r) /(1-r)) .
$$


Let $\Delta_{r}$ denote the set given by (4), $\delta_{r}$ the boundary of $\Delta_{r}$, and $\delta_{r}^{+}=\left\{W \in \delta_{r} \mid \operatorname{Im} W\right.$ $\geqslant 0\}$. If we let $g_{r}(W)$ denote the right-hand side of (3), then, since $g_{r}$ is superharmonic (in $W$ ) on $\Delta_{r}, g_{r}$ assumes its minimum over $\Delta_{r}$ on $\delta_{r}$. Further, by a symmetry argument, we can conclude that $g_{r}$ attains its minimum over $\Delta_{r}$ on $\delta_{r}^{+}$.

Let $r_{0}$ be defined by

$$
r_{0}=\max \left\{r\left|\min _{\delta_{r}^{+}} \operatorname{Re} \frac{W}{1+W}-2 r\right| \frac{W}{1+W} \mid \geqslant 0\right\} .
$$

It follows then that $r_{S_{t}}[\mathcal{L}(\delta)] \geqslant r_{0}$. We determine $r_{0}$ as follows: Line 5 implies

$$
\operatorname{Re} V-2 r_{0}|V| \geqslant 0
$$

where $V=W /(1+W), W \in \delta_{r_{0}}^{+}$. Let $V=x+i y$. Then (6) implies $x \geqslant 0$ and $0 \leqslant y \leqslant x / A$, where $A=\sqrt{4 r_{0}^{2} /\left(1-4 r_{0}^{2}\right)}$. Since the map $W \rightarrow V$ is a bilinear map with pole at $-1, W$ must be on or outside the circle $|W-C|=|C|$, where $C=-\frac{1}{2}+\frac{1}{2} A i$. Hence, if we write $W=\rho e^{i \theta}$ and use the geometry imposed on $W$, we obtain, upon simplification,

$$
\rho+\cos \theta-A \sin \theta \geqslant 0 .
$$

Let $d(\rho, \theta)$ denote the left-hand side of (7). Now, $W$ is constrained to be on $\delta_{r_{0}}^{+}$. We note for $W$ on $\delta_{r_{0}}^{+}$and for $\rho \geqslant 1$ that $\theta$ decreases with increasing $\rho$ and $d(\rho, \theta)$ increases with $\rho$. Thus, to find the minimum of $d(\rho, \theta)$, we may constrain $W$ to be on $\delta_{r_{0}}^{+}$with $\rho \leqslant 1$. For $\rho \leqslant 1$, the arc $\delta_{r_{0}}^{+}$is monotonic; therefore, $\rho$ may be written in terms of $\theta$ as $\rho=\rho(\theta)=\exp \left(-\sqrt{B^{2}-\theta^{2}}\right)$, where $B=\log \left(\left(1+r_{0}\right) /\left(1-r_{0}\right)\right)$ and $0 \leqslant \theta \leqslant B$.

Let $d(\rho, \theta)$ achieve its minimum on $\delta_{r_{0}}^{+}$at $\theta_{0}$. Then by (5) we have

$$
\rho_{0}+\cos \theta_{0}-A \sin \theta_{0}=0
$$

where $\rho_{0}=\rho\left(\theta_{0}\right)$. Further, since at $\theta_{0}$ we have a minimum of $d(\rho, \theta)$, we also have

$$
\rho_{0}^{\prime}-\sin \theta_{0}-A \cos \theta_{0}=0,
$$

where

$$
\rho_{0}^{\prime}=\rho_{0} \theta_{0} / \sqrt{B^{2}-\theta_{0}^{2}} .
$$

Multiplying ( 8 ) and (9) by $\sin \theta_{0}$ and $\cos \theta_{0}$ (and vice versa) and then adding and subtracting the resulting equations yields

$$
\rho_{0} \sin \theta_{0}+\rho_{0}^{\prime} \cos \theta_{0}-A=0,
$$

and

$$
\rho_{0} \cos \theta_{0}-\rho_{0}^{\prime} \sin \theta_{0}+1=0,
$$

which combine to give

$$
\rho_{0}\left(\sin \theta_{0}+A \cos \theta_{0}\right)+\rho_{0}^{\prime}\left(\cos \theta_{0}-A \sin \theta_{0}\right)=0 .
$$

Substituting (10) into (11) and rearranging yields

$$
\frac{\tan \theta_{0}+A}{A \tan \theta_{0}-1}=\frac{\theta_{0}}{\sqrt{B^{2}-\theta_{0}^{2}}} \text {. }
$$


Let $A=\tan \omega$. Then, rewriting (12), squaring and simplifying we have

$$
1+\tan ^{2}\left(\theta_{0}+\omega\right)=B^{2} /\left(B^{2}-\theta_{0}^{2}\right),
$$

which can be reduced to

$$
\theta_{0}=B \sin \left(\theta_{0}+\omega\right) \text {. }
$$

Equation (13) defines $\theta_{0}$ implicitly in terms of $r_{0}$. Then $r_{0}$ is found as the first positive root of (8).

In order to find $r_{0}$ we will show that $d\left(\rho_{0}, \theta_{0}\right)$ has only one zero on $0<r_{0}<.44$. Then we may apply Newton's method to obtain $.435659<r_{0}<.435660$. (For convenience, we will drop the subscript on $r_{0}$.) The critical step in the argument is to show that $\theta$ is an increasing function of $r$ on $0<r<.44$. Differentiating (13) implicitly we have

$$
\theta^{\prime}=\frac{B^{\prime} \sin (\theta+\omega)+B \cos (\theta+\omega) \omega^{\prime}}{1-B \cos (\theta+\omega)}=\frac{N(r)}{D(r)} .
$$

We see that $D(r) \geqslant 1-B>1-B(.44)>0$ on $0<r<.44$. We will show that if $N(\hat{r})=0$, then $\hat{r}>.44$. That will imply $\theta^{\prime}>0$ on $0<r<.44$ and, hence, $\theta$ is increasing on $0<r<.44$.

Suppose $N(\hat{r})=0$. That implies $\tan (\theta+\omega)=-B \omega^{\prime} / B^{\prime}$, which implies, since $\tan \omega=A$,

$$
\tan \theta=\frac{B \omega^{\prime}+A B^{\prime}}{B^{\prime}-A B \omega^{\prime}}=-\frac{N_{1}(\hat{r})}{D_{1}(\hat{r})}
$$

If $0<r<.44$, then $0 \leqslant \tan \theta \leqslant \tan B(.44)<1.39$, since $0 \leqslant \theta \leqslant B$. It is easily seen that $N_{1}(r)$ is increasing and positive on $0<r<.44$ and $D_{1}(r)$ is decreasing on $0<r<.44$ and changes sign once, say at $r_{*}$. Then, for $0<r<r_{*},-N_{1}(r) / D_{1}(r)<$ 0 , whereas $\tan \theta>0$. For $r_{*}<r<.44$, we have $(d / d r)\left(-N_{1}(r) / D_{1}(r)\right)<0$, which implies $-N_{1}(r) / D_{1}(r)>-N_{1}(.44) / D_{1}(.44)>1.75$, whereas $\tan \theta<1.39$. Therefore, $\hat{r}>.44$.

To show that $d(\rho, \theta)$ has the required properties, write

$$
\begin{aligned}
d(\rho, \theta) & =\rho+\cos \theta-A \sin \theta \\
& =\exp (-B|\cos (\theta+\omega)|)+\sqrt{1-4 r^{2}} \cos (\theta+\omega) .
\end{aligned}
$$

Clearly, $\theta+\omega$ is an increasing function on $0<r<.44$. Let $r_{1}$ satisfy $\theta\left(r_{1}\right)+\omega\left(r_{1}\right)$ $=\pi / 2$. Then, for $0<r<r_{1}, d(\rho, \theta)>\sqrt{1-4 r^{2}} \cos (\theta+\omega)>0$, and for $r_{1}<r<$ $.44, d(\rho, \theta)$ is decreasing.

REMARK. The author has not been able to determine whether the lower bound of $r_{0}$ given here for $r_{\delta_{t}}[\mathcal{L}(\mathcal{\delta})]$ is sharp. To show sharpness it is necessary (and sufficient) to show that if $f_{0}$ is the Jenkin's function (for which $z f^{\prime}{ }_{0} / f_{0}(z)$ lies on $\delta_{r_{0}}^{+}$) which minimizes the right-hand side of (3) at $|z|=r_{0}$, then

$$
\operatorname{Im}\left\{\frac{2+z f_{0}^{\prime \prime}(z) / f_{0}^{\prime}(z)}{1+f_{0}(z) /\left(z f_{0}^{\prime}(z)\right)}-\frac{2}{1-r_{0}^{2}} \frac{z f_{0}^{\prime}(z) / f_{0}(z)}{1+z f_{0}^{\prime}(z) / f_{0}(z)}\right\}=0 .
$$


EXAMPLE. Let $f_{x, y},|x|=|y|=1$, be given by

$$
f_{x, y}(z)=\left(z-\frac{1}{2}(x+y) z^{2}\right) /(1-y z)^{2} .
$$

It is well known that $f_{x, y} \in \mathcal{C}$ and, for $x \neq y, f$ maps $\mathscr{D}$ to the complement of a half-line. Let $g=\mathcal{L}\left(f_{x, y}\right)$. Then

$$
\frac{z g^{\prime}(z)}{g(z)}=\left(\frac{3}{1-y z}-\frac{1}{1-x z}\right) /\left(1+\frac{1}{2\left[\frac{1}{1-y z}-\frac{1}{1-x z+1-y z}\right]}\right) \text {. }
$$

It is easily verified that if $\arg x=343^{\circ}$ and $\arg y=100^{\circ}$, then $\operatorname{Re} z g^{\prime}(z) / g(z)<0$ for $z=r \geqslant .4447$.

\section{REFERENCES}

1. H. S. Al-Amiri, On the radius of univalence of certain analytic functions, Colloq. Math 28 (1973), 133-139.

2. On the radius of starlikeness of certain analytic functions, Proc. Amer. Math. Soc. 42 (1974), 466-474.

3. P. L. Bajpai and P. Singh, The radius of starlikeness of certain analytic functions, Proc. Amer. Math. Soc. 44 (1974), 395-402.

4. R. W. Barnard, On the radius of starlikeness of $(z f)^{\prime}$ for $f$ univalent, Proc. Amer. Math. Soc. 53 (1975), 385-390.

5. On Robinson's $\frac{1}{2}$ conjecture, Proc. Amer. Math. Soc. 72 (1978), 135-139.

6. R. W. Barnard and C. Kellog, Applications of convolution operators to problems in univalent function theory, Michigan Math. J. 27 (1980), 81-94.

7. S. Chandra and P. Singh, Certain subclasses of the class of functions regular and univalent in the unit disc, Arch. Math. 26 (1975), 60-63.

8. J. A. Jenkins, Univalent functions and conformal mapping, Springer-Verlag, Berlin and New York, 1965.

9. R. J. Libera and A. E. Livingston, On the univalence of some classes of regular functions, Proc. Amer. Math. Soc. 30 (1971), 327-336.

10. A. E. Livingston, On the radius of univalence of certain analytic functions, Proc. Amer. Math. Soc. 17 (1966), 352-357.

11. K. S. Padmanabhan, On the radius of univalence of certain classes of analytic functions, J. London Math. Soc. (2) 1 (1969), 225-231.

12. R. M. Robinson, Univalent majorants, Trans. Amer. Math. Soc. 61 (1947), 1-35.

Department of Mathematics, TeXas Tech University, Lubbock, TeXas 79409 